\title{
Letters
}

\section{Evaluation of the Radar Cross Section of Circular Microstrip Patches on Anisotropic and Chiral Substrates}

\author{
V. Losada, R. R. Boix, and F. Medina
}

\begin{abstract}
Galerkin's method in the Hankel transform domain (HTD) is applied to the determination of the radar cross section (RCS) of a circular microstrip patch printed on a substrate which may be an uniaxial anisotropic dielectric, a magnetized ferrite, or a chiral material. The results obtained for circular patches on magnetized ferrites show that the RCS of these patches can be substantially reduced in a tunable frequency band when a bias magnetic field is applied. It is also shown that the results obtained for the RCS of circular patches printed on chiral materials can be substantially different from those obtained when substrate chirality is ignored.
\end{abstract}

Index Terms-Complex media, microstrip patches, scattering.

\section{INTRODUCTION}

As the radar cross section (RCS) of a military platform is reduced by geometrical shaping and the use of composite radar absorbing materials, it becomes increasingly important to consider the RCS of antennas mounted on the whole structure since the scattering from these antennas may give the most important contribution to the overall RCS of a low-observable platform. Both theoretical and experimental results for the RCS of circular and rectangular microstrip antennas on isotropic dielectric substrates have been obtained and published in [1], [2]. Also, Pozar has studied how the RCS of rectangular microstrip antennas is affected by the use of anisotropic dielectric substrates [3] and anisotropic magnetic substrates [4], [5]. The performance of microstrip antennas fabricated on chiral substrates has been analyzed in [6], [7], but as far as the authors know, the scattering properties of these antennas have not been studied in detail. In this paper the authors apply Galerkin's method in the Hankel transform domain (HTD) [8] to the determination of the RCS of unloaded microstrip patch antennas of circular shape fabricated on a substrate which may be an uniaxial anisotropic dielectric, a magnetized ferrite, or a chiral material. In particular, emphasis is placed on analyzing how the scattering properties of circular microstrip patches are modified when the conventional isotropic dielectric substrates are substituted by nonconventional substrates such as anisotropic dielectrics, magnetized ferrites and chiral materials.

\section{Determination of the RCS OF THE CiRCUlar Patch}

Fig. 1 shows the side and top views of a circular microstrip patch of radius $a$. Both the circular metallic patch and the ground plane of Fig. 1 are assumed to be perfect electric conductors (PEC) of negligible thickness. The substrate is assumed to be of infinite extent along the $x$ and $y$ coordinates and this substrate is assumed to be made of any of the three following materials: a uniaxial anisotropic dielectric, a normally

Manuscript received October 15, 1999; revised February 6, 2001. This work was supported by the CICYT, Spain, Project TIC98-0630.

V. Losada is with the Microwaves Group, Department of Applied Physics, E. U. I. T. A. University of Seville, 41013, Sevilla, Spain.

R. R. Boix and F. Medina are with the Microwaves Group, Department of Electronics and Electromagnetism, School of Physics, University of Seville, 41012 Sevilla, Spain (e-mail: boix @cica.es).

Publisher Item Identifier S 0018-926X(01)06377-3.
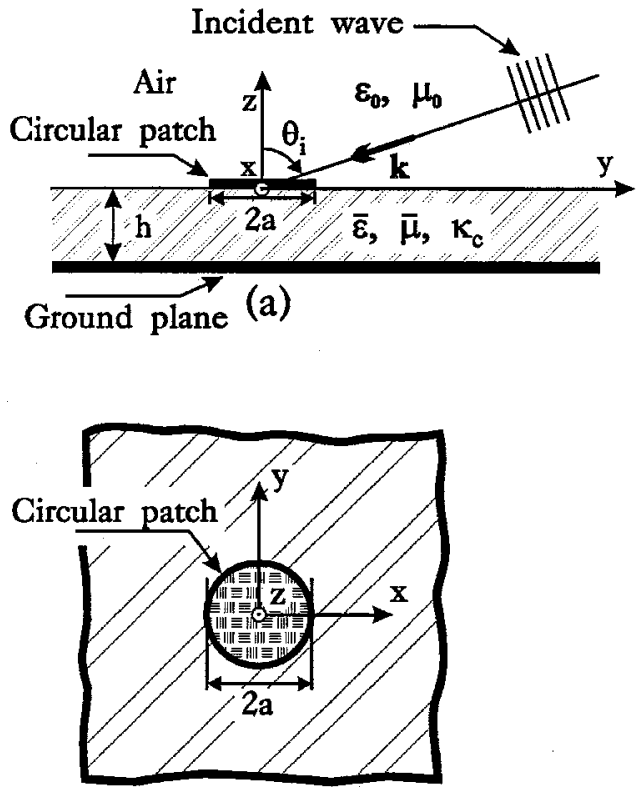

(b)

Fig. 1. Side (a) and top (b) views of a circular microstrip patch printed on a substrate which may be an uniaxial anisotropic dielectric, a magnetized ferrite, or a chiral material. A plane wave whose direction of propagation makes an angle $\theta_{i}$ with the $z$-axis incides on the circular microstrip patch.

biased ferrite, and a chiral material. In case the substrate is a uniaxial anisotropic dielectric, it will be assumed that its permeability is $\mu_{0}$ and the permittivity tensor is of the form [3]

$$
\overline{\boldsymbol{\epsilon}}=\epsilon_{0}\left(\begin{array}{ccc}
\epsilon_{x} & 0 & 0 \\
0 & \epsilon_{x} & 0 \\
0 & 0 & \epsilon_{z}
\end{array}\right) .
$$

In case the substrate is a magnetized ferrite, it will be assumed that the bias magnetic field is directed along the $z$-axis of Fig. 1. For that case, the permittivity of the ferrite material will be taken as $\epsilon_{0} \epsilon_{f}$ and the permeability tensor will be taken as [4]

$$
\overline{\boldsymbol{\mu}}=\mu_{0}\left(\begin{array}{ccc}
\mu & j \kappa & 0 \\
-j \kappa & \mu & 0 \\
0 & 0 & 1
\end{array}\right)
$$

where the elements of the permeability tensor $\bar{\mu}$ can be obtained in terms of the gyromagnetic ratio $\gamma=1.759 \cdot 10^{11} \mathrm{C} / \mathrm{Kg}$, the saturation magnetization of the ferrite material $M_{s}$, the internal bias magnetic field $H_{0}$ and the linewidth $\Delta H$ as explained in [9].

Finally, in case the substrate of Fig. 1 is a chiral material, this material will be assumed to be a Pasteur medium [10] with permittivity $\epsilon_{0} \epsilon_{c}$, permeability $\mu_{0} \mu_{c}$ and dimensionless chiral parameter $\kappa_{c}$. Inside this material, the constitutive relations among the four vector quantities $\mathbf{D}, \mathbf{B}, \mathbf{E}$, and $\mathbf{H}$ will be given by [10]

$$
\begin{aligned}
& \mathbf{D}=\epsilon_{0} \epsilon_{c} \mathbf{E}-j \kappa_{c} \sqrt{\epsilon_{0} \mu_{0}} \mathbf{H} \\
& \mathbf{B}=\mu_{0} \mu_{c} \mathbf{H}+j \kappa_{c} \sqrt{\epsilon_{0} \mu_{0}} \mathbf{E} .
\end{aligned}
$$

Let us assume that a TEM plane wave traveling through the air incides on the circular microstrip patch of Fig. 1. This plane wave im- 
pinging on the structure of Fig. 1 will be both reflected by the substrate and scattered by the circular metallic patch. The electric field of the wave reflected by the substrate in the absence of the patch can be obtained in terms of the electric field of the incident wave as explained in [11]. The electric field scattered by the patch can be expressed in terms of the induced current density by means of a suitable dyadic Green's function (see [8, eq. (1)]). If the total tangential electric field on the patch (sum of the electric fields of the incident wave, the reflected wave and the scattered wave) is obliged to be zero, an electric field integral equation (EFIE) for the induced current density on the patch is obtained (see [1, eq. (3)]). In order to solve this EFIE, in the current paper the authors have expressed the unknown current density on the patch in cylindrical coordinates - $\mathbf{j}=\mathbf{j}(\rho, \phi)$-as a Fourier series of the cylindrical coordinate $\phi$ given by

$$
\begin{aligned}
\mathbf{j}(\rho, \phi) & =\sum_{m=-\infty}^{+\infty} \mathbf{j}_{m}(\rho) e^{j m \phi} \\
& =\sum_{m=-\infty}^{+\infty}\left(j_{m, \rho}(\rho) \hat{\boldsymbol{\rho}}+j_{m, \phi}(\rho) \hat{\boldsymbol{\phi}}\right) e^{j m \phi} .
\end{aligned}
$$

Bearing in mind (5), the original EFIE has been written in the HTD in order to obtain an infinite set of decoupled equations for the Hankel transforms of the vector functions $\mathbf{j}_{m}(\rho)$ appearing in the modal decomposition of $\mathbf{j}(\rho, \phi)$. Each of these decoupled equations has been solved by applying Galerkin method in the HTD as in [8]. The basis functions for $j_{m, \rho}(\rho)$ and $j_{m, \phi}(\rho)$ have been chosen to be those shown in [12, eqs. (10)-(13)]. The dyadic Green's function of the substrate in the HTD has been obtained in terms of its two-dimensional (2-D)Fourier transform as explained in [8]. Once the different functions $\mathbf{j}_{m}(\rho)$ of (5) have been calculated, the far zone scattered electric field $\mathbf{E}_{s c}^{f f}(r, \theta, \phi)$ has been computed in terms of the Hankel transforms of $\mathbf{j}_{m}(\rho)$ by applying the stationary phase method (see [8, eqs. (23), (24)]). Finally, the different components of the monostatic radar cross section of the circular patch of Fig. 1 have been obtained in terms of $\mathbf{E}_{s c}^{f f}$ as

$$
\sigma_{u v}^{m s}=\frac{4 \pi r^{2}\left|\mathbf{E}_{s c}^{f f}\left(r, \theta=\theta_{i}, \phi=\phi_{i}\right) \cdot \hat{v}\right|^{2}}{\left|E_{u}^{i}\right|^{2}} \quad(u, v=\theta, \phi)
$$

where $\mathbf{E}_{s c}^{f f} \cdot \hat{\boldsymbol{v}}$ stands for the $v$ component of the scattered electric field $(v=\theta, \phi)$ and $\left|E_{u}^{i}\right|$ stands for the magnitude of the electric field of an incident plane wave polarized in the $u$ direction $(u=\theta, \phi)$.

\section{NUMERICAL RESULTS}

In order to check the validity of the RCS computation algorithm described in Section II, the numerical and experimental results published [2, Fig. 7] for the RCS of a circular microstrip patch printed on an isotropic dielectric have been compared with numerical results obtained via the aforementioned algorithm. Excellent agreement has been found among the three sets of results.

Fig. 2 presents results for the copolarized RCS component $\sigma_{\theta \theta}^{m s}$ of a circular microstrip patch printed on an anisotropic dielectric substrate (pyrolitic boron nitride). The cross-polarized component $\sigma_{\theta \phi}^{m s}$ is zero as it happens when the substrate is an isotropic dielectric. It should be pointed out that in the range of frequencies studied in Fig. 2 the authors have found that the use of nine terms in the series of (5) (namely, $m=$ $-4,-3,-2,-1,0,1,2,3,4)$ and the use of seven basis functions in the approximation of every vector function $\mathbf{j}_{m}(\rho)$ suffice to obtain convergent results for the RCS within four significant figures. The resonant frequencies indicated by the arrows of Fig. 2 have been obtained by using the method reported in [12]. In Fig. 2 the results obtained for

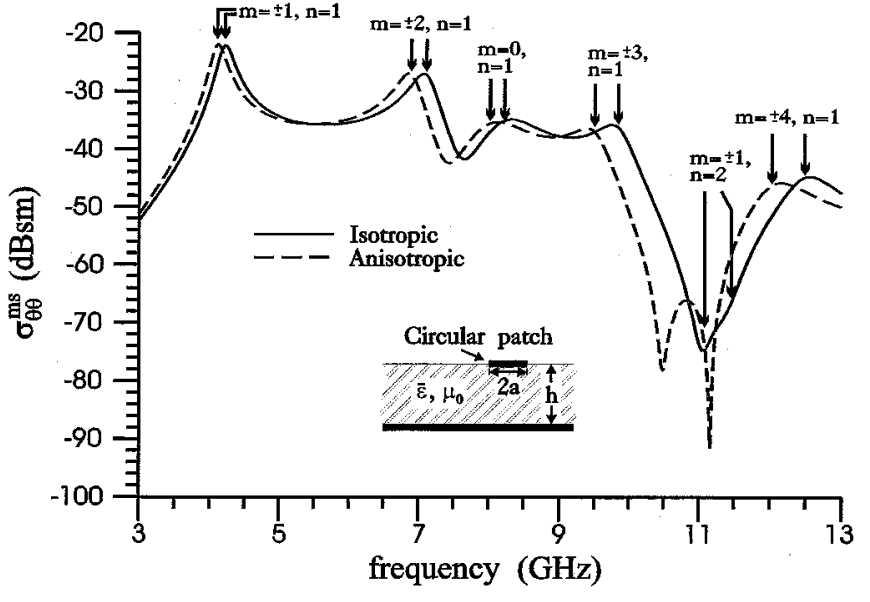

Fig. 2. Monostatic RCS component $\sigma_{\theta \theta}^{m s}$ of a circular microstrip patch versus frequency $\left(\theta_{i}=60^{\circ}, a=10.16 \mathrm{~mm}\right)$. The substrate is taken to be either anisotropic P.B.N. $\left(h=2.54 \mathrm{~mm}, \epsilon_{x}=5.12, \epsilon_{z}=3.4\right)$ or isotropic P.B.N. ( $\left.h=2.54 \mathrm{~mm}, \epsilon_{x}=\epsilon_{z}=3.4\right)$. Vertical arrows locate the resonant frequencies of the circular patch.
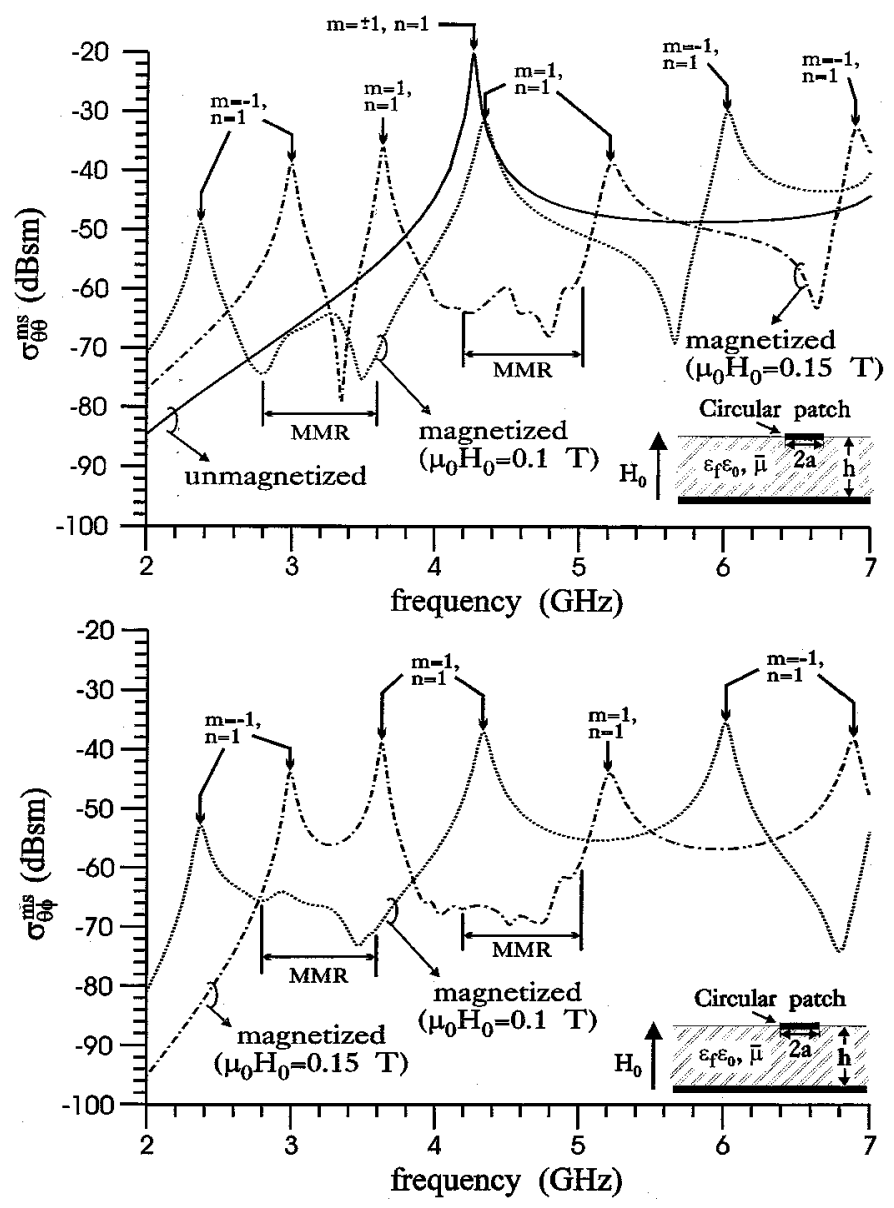

Fig. 3. Monostatic RCS components $\sigma_{\theta \theta}^{m s}$ (a) and $\sigma_{\theta \phi}^{m s}$ (b) of a circular microstrip patch versus frequency $\left(\theta_{i}=60^{\circ}, a=5 \mathrm{~mm}\right)$. The substrate is taken to be a ferrite material $\left(h=1.27 \mathrm{~mm}, \epsilon_{f}=15\right)$ which can be unmagnetized $\left(M_{s}=H_{0}=\Delta H=0\right)$ or magnetically biased $\left(\mu_{0} M_{s}=0.065 \mathrm{~T}, H_{0} \neq 0, \mu_{0} \Delta H=0.004 \mathrm{~T}\right)$. MMR is the acronym for the magnetostatic mode regions.

the circular microstrip patch printed on anisotropic dielectric are compared with the results that would be obtained if dielectric anisotropy were neglected. It can be noticed that although the differences between 

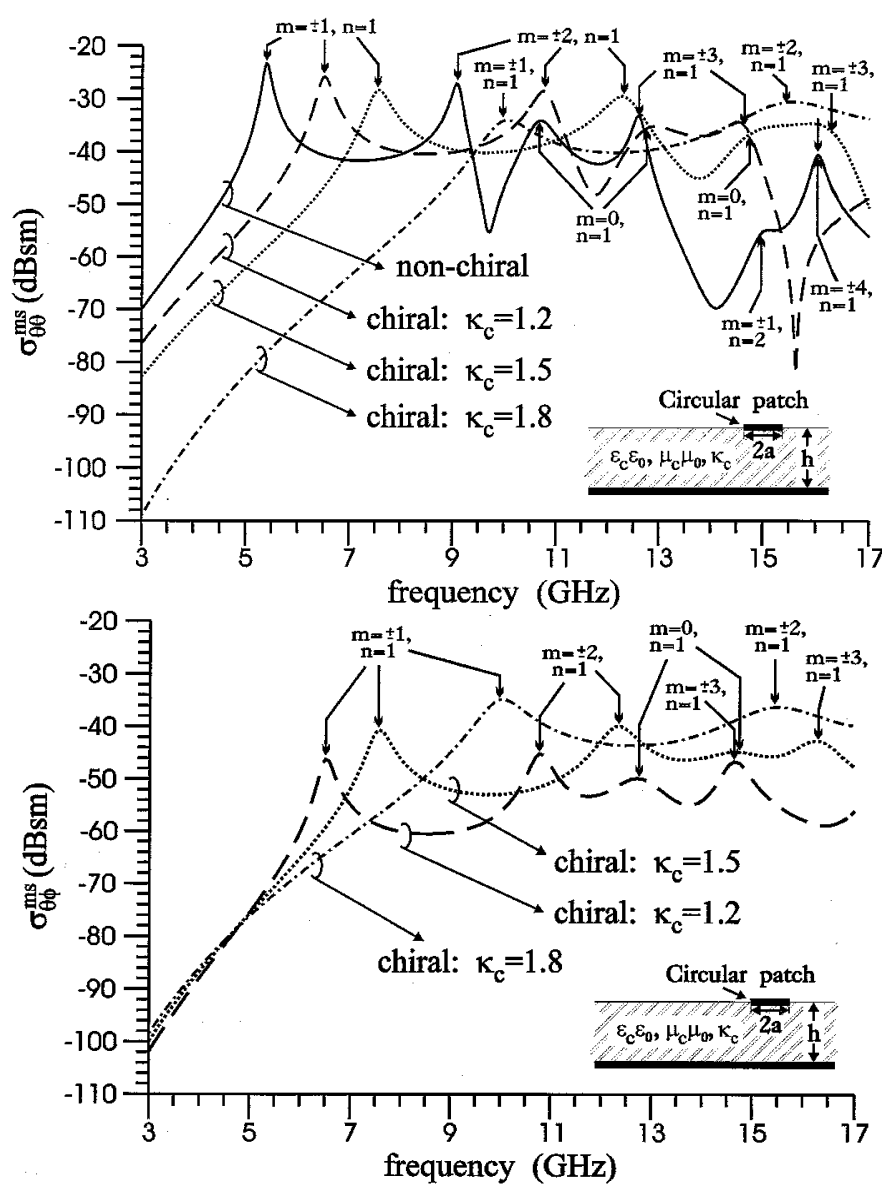

Fig. 4. Monostatic RCS components $\sigma_{\theta \theta}^{m s}$ (a) and $\sigma_{\theta s}^{m s}$ (b) of a circular microstrip patch versus frequency $\left(\theta_{i}=60^{\circ}, a=7.62 \mathrm{~mm}\right)$. The substrate is taken to be either a nonchiral dielectric material $\left(h=1.27 \mathrm{~mm}, \epsilon_{c}=4\right.$, $\mu_{c}=1, \kappa_{c}=0$ ) or a set of chiral materials with different values of $\kappa_{c}$ $\left(h=1.27 \mathrm{~mm}, \epsilon_{c}=4, \mu_{c}=1\right)$.

the results obtained considering dielectric anisotropy and neglecting dielectric anisotropy increase with increasing frequency, these differences never reach very high values. So, the differences between the frequencies of the resonant peaks are always within $4 \%$ and the differences between the levels of these resonant peaks are always within 2.5 dBsm.

In Fig. 3(a) and (b) results are presented for the RCS components $\sigma_{\theta \theta}^{m s}$ and $\sigma_{\theta \phi}^{m s}$ of a microstrip patch on a ferrite substrate. The peaks of the cross-polarized component $\sigma_{\theta \phi}^{m s}$ are on average $6 \mathrm{dBsm}$ below those of the copolarized component $\sigma_{\theta \theta}^{m s}$. Fig. 3(a) and (b) show that as the ferrite substrate is switched from the unmagnetized to the magnetized state, the two components of the RCS are considerably reduced (below $-60 \mathrm{dBsm})$ in the whole frequency band in which magnetostatic volume-wave modes can propagate along the ferrite substrate, i.e., in the frequency band $\mu_{0} \gamma H_{0} / 2 \pi<f<\mu_{0} \gamma \sqrt{H_{0}\left(H_{0}+M_{s}\right)} / 2 \pi$. In order to explain this RCS reduction, one has to take into account that the magnetostatic mode frequency region is a cutoff frequency region in which resonances of the circular patch on normally biased ferrite cannot occur owing to the excitation in that frequency region of an infinite number of magnetostatic volume-wave modes along the conductor-backed ferrite layer [12]. Then, the results of Fig. 3(a) and (b) indicate that microstrip patches fabricated on normally biased ferrites present a whole frequency band with very low values of RCS and that this frequency band can be tuned by adjusting the magnitude of the bias magnetic field [compare the results of Fig. 3(a) and (b) for $\mu_{0} H_{0}=0.1 \mathrm{~T}$ and $\left.\mu_{0} H_{0}=0.15 \mathrm{~T}\right]$.
In Fig. 4(a) and (b) the results obtained for the RCS components $\sigma_{\theta \theta}^{m s}$ and $\sigma_{\theta \phi}^{m s}$ of a circular microstrip patch printed on different chiral substrates are compared with the results obtained when the circular patch is printed on a nonchiral dielectric substrate of the same permittivity as that of the chiral substrates. It can be noticed that the cross-polarization increases as $\kappa_{c}$ increases (whereas the peaks of $\sigma_{\theta \phi}^{m s}$ are on average 17 dBsm below those of $\sigma_{\theta \theta}^{m s}$ when $\kappa_{c}=1.2$, this difference reduces to $10 \mathrm{dBsm}$ when $\kappa_{c}=1.5$, and to $3 \mathrm{dBsm}$ when $\kappa_{c}=1.8$ ), which is in agreement with the results obtained in [6]. Fig. 4(a) and (b) show that the differences between the frequencies of the RCS peaks obtained in the chiral case and those obtained in the nonchiral case increase as $\kappa_{c}$ increases (in fact, these differences are around $18 \%$ when $\kappa_{c}=1.2$, around $35 \%$ when $\kappa_{c}=1.5$, and around $80 \%$ when $\kappa_{c}=1.8$ ). Also, the RCS peaks of the patch on chiral substrates tend to be lower and smoother as $\kappa_{c}$ increases, which can be explained by considering that the quality factors of the resonant modes of the patch on chiral substrates decrease as the chiral parameter $\kappa_{c}$ increases [12].

\section{CONCLUSION}

Galerkin's method in the HTD has been used for the numerical determination of the RCS of a circular microstrip patch printed on a substrate which may be an uniaxial anisotropic dielectric, a magnetized ferrite, or a chiral material. It has been shown that the RCS of a microstrip patch on a magnetized ferrite with bias magnetic field can be substantially reduced in a tunable frequency band, which is attributed to the excitation of an infinite number of magnetostatic volume-wave modes along the ferrite substrate in that frequency band. Also, the results obtained show that whereas small errors are made in the computation of the RCS when substate dielectric anisotropy is ignored, very large errors may be made in case substrate chirality is ignored.

\section{REFERENCES}

[1] E. H. Newman and D. Forrai, "Scattering from a microstrip patch," IEEE Trans. Antennas Propagat., vol. AP-35, pp. 245-251, Mar. 1987.

[2] J. T. Aberle, D. M. Pozar, and C. R. Bitcher, "Evaluation of input impedance and radar cross section of probe-fed microstrip patch elements using an accurate feed model," IEEE Trans. Antennas Propagat., vol. 39, pp. 1691-1696, Dec. 1991.

[3] D. M. Pozar, "Radiation and scattering from a microstrip patch on a uniaxial substrate," IEEE Trans. Antennas Propagat., vol. AP-35, pp. 613-621, June 1987.

[4] - "Radiation and scattering characteristics of microstrip antennas on normally biased ferrite substrates," IEEE Trans. Antennas Propagat., vol. 40, pp. 1084-1092, Sept. 1992.

[5] D. M. Pozar and H. Y. Yang, "Correction to 'Radiation and scattering characteristics of microstrip antennas on normally biased ferrite substrates'," IEEE Trans. Antennas Propagat., vol. 42, pp. 122-123, Jan. 1994.

[6] D. M. Pozar, "Microstrip antennas and arrays on chiral substrates," IEEE Trans. Antennas Propagat., vol. 40, pp. 1260-1263, Oct. 1992.

[7] A. Toscano and L. Vegni, "A new efficient moment method formulation for the design of microstrip antennas over a chiral grounded slab," $J$. Electromagn. Waves Appl., vol. 11, no. 5, pp. 567-592, 1997.

[8] V. Losada, R. R. Boix, and M. Horno, "Resonant modes of circular microstrip patches in multilayered substrates," IEEE Trans. Microwave Theory Tech., vol. 47, pp. 488-498, April 1999.

[9] D. M. Pozar, Microwave Engineering. Reading, MA: Addison-Wesley, 1990.

[10] I. V. Lindell, A. H. Sihvola, S. A. Tretyakov, and A. J. Viitanen, Electromagnetic Waves in Chiral and Bi-Isotropic Media. Norwood, MA: Artech House, 1994.

[11] J. L. Tsalamengas, "Interaction of electromagnetic waves with general bianisotropic slabs," IEEE Trans. Microwave Theory Tech., vol. 40, pp. 1870-1878, Oct. 1992.

[12] V. Losada, R. R. Boix, and M. Horno, "Full-wave analysis of circular microstrip resonators in multilayered media containing uniaxial anisotropic dielectrics, magnetized ferrites, and chiral materials," IEEE Trans. Microwave Theory Tech., vol. 48, pp. 1057-1064, June 2000. 